\title{
Using SSM to Rethink the Analysis of Energy Efficiency Initiatives
}

\author{
Luís Miguel Pires Neves \\ School of Technology and Management, Polytechnic Institute of Leiria; INESC Coimbra, Portugal \\ António Gomes Martins \\ Carlos Henggeler Antunes \\ Department of Electrical Engineering and Computers, University of Coimbra; INESC Coimbra, Portugal \\ Luís Cândido Dias \\ Faculty of Economics, University of Coimbra; INESC Coimbra, Portugal
}

Running head: SSM to Rethink Energy Efficiency

Keywords: Energy; Systems; Soft OR;

\begin{abstract}
This paper reflects an attempt to rethink the process of analysis of Energy Efficiency Initiatives using Soft Systems Methodology (SSM) as a problem structuring tool. The aim of the work is to provide public and private initiative promoters or evaluators with a structured support for a more informed decision, regarding the implementation of energy efficiency measures. The SSM approach contributed with the identification of all market players and their relations, as well as the insight into the deficiencies of current methodologies. Some future work directions are also proposed.
\end{abstract}

\section{Introduction}

Energy Efficiency of end-uses has been naturally increasing in the last decades, at the pace of technology innovation and transfer, although in a much lower scale than its potential. This is a well-known problem, often 
mentioned by politicians, when considering policy options for reducing environmental damage and the dependence of countries regarding external energy supply.

In the past, electric utilities involved themselves in Demand-Side Management (DSM) programmes, trying to stimulate consumers to adopt a more convenient consumption pattern, including conservation measures. In fact, DSM could be a business opportunity, and a way of avoiding increasing marginal costs caused by the oil crisis of the seventies and eighties.

In the US, these programmes spread until the middle of the nineties, being financed by a general increase in tariffs, allowed by the regulators. In this context, electric utilities had to demonstrate the cost-effectiveness of DSM programmes, according to the definitions imposed by the Public Utilities Commissions (PUC).

With the reduction of energy (oil) prices and the restructuring of the Electricity Market, new ways of fostering energy efficiency had to be found, as utilities lost part of the motivation and means. The Market Transformation concept has then emerged, with governments and other entities directly promoting efficiency with the ambition of transforming the market on a more permanent basis.

The way of analysing the proposed initiatives was adapted from the DSM framework, the focus being given to the societal perspective, with the environmental impact, and particularly the greenhouse effect, as the main concerns. However, the result of this adaptation may well be considered unsatisfactory for providing adequate decision support. Indeed, this way of analysing energy efficiency initiatives raises a few questions:

Can one be sure of the value assigned to issues such as tons of avoided $\mathrm{CO}_{2}$, or to health benefits from improved living conditions? Can one determine how much $\mathrm{CO}_{2}$ is actually avoided by an increase in energy efficiency?

How can one compare two initiatives by their Net Present Value (NPV) of benefits without considering the inevitable tradeoffs hidden behind or ignored by this indicator, specially when different perspectives are involved such as those of consumers, society, energy companies?

The work currently under way aims at devising a new way of deciding the interest in promoting, financing or supporting one initiative or a set of initiatives. 
The pursuit of this objective requires a novel approach capable of encompassing the whole problem context and structuring the problematic issues to be faced by different players. Soft Systems Methodology was used to explore the situation, identifying the stakeholders and their relations, and modelling the evaluation system to be used by an interested party, questioning the pure quantitative approach embodied in cost-benefit analysis. Moreover, it helps to discuss whether sheer quantitative tools can satisfactorily tackle complex issues. This work brings SSM to a new and important area of application characterised by a mesh of problematic issues which require a structuring tool, laying the foundations for the development of decision support systems based on multi-criteria decision analysis (MCDA). The main benefits regarding the relationships between SSM and MCDA lie on the SSM capabilities to raise all the issues of distinct nature that must be taken into account in multi-criteria models ${ }^{1}$. The idea of using problem structuring methods (Soft OR) for deriving criteria to be used in MCDA is not new, although previous research has focused on cognitive mapping rather than $\mathrm{SSM}^{2 / 3}$.

This paper firstly presents the current status of Energy Efficiency Initiatives analysis. Secondly, it explains the reason for the choice of Soft Systems Methodology as a problem structuring tool, the following section describing its application to the problem. Finally, some conclusions are drawn about the proposed methodology of analysing energy efficiency, namely regarding the application of SSM. Some directions of future work are also outlined.

\section{Current status of Energy Efficiency Initiatives Analysis}

In 1983, when Load Management initiatives were being tested by US Electric Utilities, the California Public Utilities Commission (CPUC) and the California Energy Commission (CEC) published the "Standard Practice for Cost-Benefit Analysis of Conservation and Load Management Programs, 4 . This manual introduced a set of standard Cost-Benefit tests to be used by these commissions in the evaluation of the initiatives proposed by the Californian utilities.

These tests were changed in 1987 to conform to the already existent definition of DSM, and taking into account the criticisms raised against the 1983 tests. The new set of tests was defined in the "Standard Practice 
Manual: Economic Analysis of Demand-Side Management Programs ${ }^{, 5}$ and soon became of universal use.

The Standard Practice defined tests according to five perspectives: the participant customer in DSM programmes, the average rate-payer, the utility, the DSM programme as a resource, and the society as a variant of the latter perspective.

For each perspective, the manual defines cost-benefit and cost-effectiveness equations, depending on the metric chosen for the test (NPV, Benefit-Cost ratio, or a levelized cost). The Standard-Practice Manual states that the tests "are not intended to be used individually or in isolation. [..]. This multi-perspective approach will require utilities and Commissions to consider tradeoffs between the various tests". However, there is neither a specification on how these tradeoffs should be considered, nor practical evidences of the actual consideration of explicit tradeoffs. Rather, discussions focussed on which test to consider ${ }^{6}$.

The adoption of DSM by utilities in other countries, based on the US experience, led also to the adoption of the Standard-Practice tests ${ }^{7}$. However, in 1996, "A Guidebook for B/C Evaluation of DSM and Energy Efficiency Services Programs" was published, resulting from a project under the SAVE Programme, funded by the European Commission and with the participation of several European Utilities and Energy Agencies, which aimed to propose a "European B/C Analysis Methodology (EUBC), $\sqrt{8}$. This methodology tried to define methods more adapted to market conditions, utility structure and regulation of the different European Countries. The methodology includes also the consideration of new realities like deregulation and Market Transformation, non-existent at the time of publication of the California manual.

The EUBC methodology was more ambitious than the Standard-Practice, by defining several impacts that could be considered in the different perspectives without the need of monetisation, or even quantification. However, the methodology was still based on cost-benefit equations, the use of impacts not measured in currency terms being merely informative. The combined use of the results in the different perspectives is also not documented, allowing the use of a single criterion. In spite of some improvements regarding the California methodology, there is no evidence of its actual use by European utilities or regulatory agencies. Moreover, the generalised use of American software for energy planning is possibly a barrier to the use of EUBC. 
The advent of Market Transformation initiatives, which became more a public policy issue than a business issue, faded away the cost-benefit tests on perspectives other than the societal, with a major focus on the avoided carbon emissions, given current concerns on climate change and the greenhouse effect. An example of this is the Public Purpose Test, heir of the societal test, proposed in 1998 by Joe Eto et al to the California Board For Energy Efficiency as found in http://cpuc.ee.support.net/library/guide2-4.doc, accessed 8 December 2002. It includes monetised and non-monetised environmental impacts, the latter for documentation purposes only, to be used as the single way of analysing proposed initiatives funded by the Public Goods Charge which is applied in California to energy bills.

\section{Why SSM?}

In view of the questions raised in the introductory section, cost benefit or cost effectiveness analysis seem inadequate for evaluating energy efficiency initiatives. This perception led to the need of a clear formulation of the problem in order to seek better solutions.

The fact is that the concept of cost-effectiveness or cost-benefit analysis is so well established that any attempt to rethink this process falls easily into a similar approach. A complete re-statement of the problem is therefore needed so that all actors and issues are clearly identified.

The use of a problem structuring methodology emerged then as a valuable tool in this process and, from a first analysis, Soft Systems Methodology was elected, in part because the systems language was familiar to the authors' common background on electrical and computer engineering.

SSM has been used to gain insight into problematic situations, and in this case there was a need to gain insight into the problematic concept of the interest of energy efficiency initiatives.

With SSM it is possible to look at the whole context where energy efficiency initiatives are taken, defining a system for providing decision support on which initiatives are "good" and to whom, and to devise all possible implications of this decision.

The identification of the potential user of such a system is an issue which turns this approach into a non- 
traditional SSM study. Indeed, there was neither a problem-owner asking a problem-solver to perform a study nor the problem-solvers work in any of the entities that play a role in the situation. The driving idea is that the assessment of energy efficiency initiatives can be performed by more than one of these entities, the resulting system being made flexible enough to allow that an analysis methodology or even a computational tool can be developed to assist decision makers (DMs) from different entities.

The ultimate goal of this process is the formulation of a more adequate decision model, that should give DMs in different player entities a structured support for a more informed decision, regarding the implementation of energy efficiency initiatives.

\section{Rethinking the analysis process}

The application of SSM followed the classic seven stages displayed in figure 1 as found in Checkland and Scholes ${ }^{9}$ and Checkland $10[11$. The objective, as already stated, is to gain insight into the process of analysing a proposed initiative and declaring the interest of implementing it. Possible initiative promoters, sponsors, victims, beneficiaries and power relations must be considered.

\section{Capturing the situation}

The first stages of SSM application were developed according to the definitions of Analysis one, two and three, as suggested by Checkland and Scholes ${ }^{9}$.

The major outcomes were the identification of all the agents in the market, promoting or affected by energy efficiency initiatives, their role in the process, and the relations of power.

The resulting rich-picture (figure 2) shows six agents with a potential interest in a system to analyse energy efficiency initiatives. They are:

1. An Energy Agency, whose role is to manage public funds aimed at fostering energy efficiency, through its own initiatives or financing third parties. It needs to assess the actual interest of the society on such initiatives. 
2. The Energy market regulator, if energy efficiency is a key issue in the regulatory framework. The regulator can be mandated to foster efficiency (not only economic efficiency, but also energy efficiency), through tariff mechanisms, and in such a case it needs an adequate way to evaluate initiatives proposed by regulated companies.

3. The Government. Legislative measures, towards Market Transformation and to make Energy Efficiency a generalised concern, have to be considered a good way of spending tax money, assessing all societal impacts and political costs.

4. The Energy Companies. In this study the focus has been on Electric Energy, in the framework of the undergoing liberalisation process. There are four different roles, corresponding to generation, transmission, distribution and retail companies, each with a different perspective regarding energy efficiency initiatives. The assumption used in the paper is that generation companies are now too distant from end-users to have a direct interest on these initiatives. Transmission and distribution companies are regulated and may be subject to tariff mechanisms or even mandates to promote efficiency, which demands a process of analysis to decide which initiatives should be implemented. There is no significant difference between transmission and distribution companies regarding the way efficiency initiatives can be analysed. The difference exists on the kind of initiatives that can be taken. Retail companies have the commercial relation with energy consumers and, for them, energy efficiency can be seen as a business opportunity, a marketing tool or a threat.

5. The Energy Service Companies (ESCOs). Their business is energy efficiency. They may propose initiatives based on pure business analysis, or they may cooperate with other entities which in some cases may need a "system" to demonstrate the interest of initiatives.

6. The End-use equipment manufacturers. They can be forced to bring efficiency to the market through standards or mandatory labelling, or they can use energy efficiency as a marketing tool. In every case, there are reasons pro and against their involvement which are beyond pure economical ones. 
Each of these actors need to consider not only a position regarding its own initiatives but also regarding initiatives of others that may have an impact on its "life", specially if there is a relation of power with the proposer. For example, the Government may react to a initiative proposed by the Agency if the impact on tax revenue is estimated to be too high.

The rich-picture shows other actors affected by or influencing decisions, namely the affected energy consumers, environmental pressure groups and the society as a whole, but none of these entities can be seen as a user of a system of analysis, except indirectly through one of the other entities.

Some of the relations between actors can be understood from the following assumptions:

- The Energy Agency and the Regulator are public bodies, entitled by the Government with some power over the energy market.

- The Regulator has the power to define energy tariffs that the regulated companies are allowed to practice. The regulatory framework may also include energy efficiency obligations.

- Energy companies may implement energy efficiency initiatives by direct interest, for marketing purposes, taking benefit of cost-recovery schemes under the regulatory framework, or being financed by the Agency. Sometimes they can also be forced by the regulatory framework to achieve some target in terms of energy efficiency.

- Equipment manufacturers/dealers can be forced to cope with efficiency standards or labelling schemes by the government, or they can be invited to participate in specific initiatives. They can also apply for financing in order to accelerate the introduction of new technologies in the market.

- Consumers, Environmentalists and the Society as a whole have enough power to influence the decisions of all the actors, so their concerns have to be taken into account by the DM's preferences at the moment of deciding the implementation of a given initiative. 


\section{Root definitions and CATWOE analysis}

The present work seeks to characterise a system that can be used under any of the different perspectives of evaluation. The root definition of such a system is:

System which aims at evaluating the interest of promoting initiatives to foster the efficiency of energy end-uses considering the advantages and disadvantages to the promoter, as to other involved entities.

This definition can be applied to all the six perspectives referred to above. The preferences of the entity which analyses a given initiative (the DM) and the power relations with other entities have to be considered dynamically.

The CATWOE mnemonic led to the following definitions:

Customer - The initiative promoter, the external sponsor if any, the beneficiaries (the consumers who benefit with the initiative, the society as it concerns to environmental and other benefits, the manufacturers and sellers of promoted equipments, etc.) and victims (energy companies which reduce sales, manufacturers of the replaced equipments).

Actors - The DM, i.e., the promoter of the initiative, or someone who has the responsibility of evaluating it, due to some contract. One of the six entities referred to above: Energy Agency, Energy Market Regulator, the Government, Energy Companies, Energy Service Companies, Equipment Manufacturers.

Transformation - Initiative with unknown interest $\longrightarrow$ Interest known.

"Weltanschauung" - An initiative is implemented only if its advantages overwhelm its disadvantages to the promoter, including the ones resulting from the reactions of other affected entities.

Owner - The DM, or someone at a degree above in the hierarchy (the Government as the power above the Agency or the Regulator). 
Environment - Capability of obtaining relevant data; Estimation of initiative success (potential adherence of end-users); Budget; International agreements and directives.

\section{Modelling the system}

The resulting conceptual model of the system is shown in figure 3 together with a sub-system found to be necessary during the modelling phase.

Activity 1 deals with the gathering of all the data from the initiative definition: objectives, targets, implementing agents, estimated costs, estimated achievements.

Activity 2 is needed to assess which entities other than the DM are affected by the initiative in order to obtain possible reactions.

The assessment of these reactions, which is made in activity 4 , needs an estimate of the initiative success, based on the potential "participation" level of consumers obtained in activity 3. This input is also necessary for the correct computation of all the effects of the initiative in activity 6.

In certain cases, the available budget for implementing or sponsoring the initiative may be an important issue, its evaluation in activity 5 providing important information to activity 7.

The "comparison" of all the advantages and disadvantages to the DM made in activity 7 is the objective of this system and it emphasises the need of a multi-criteria decision aid tool.

The monitoring and control activities that are generally included in any SSM model imply the definition of criteria for assessing:

Efficacy - Does the system identify correctly a valid energy efficiency initiative?

Efficiency - Does the system work with the minimum resources?

Effectiveness - Do initiatives well classified by the system actually get implemented?

For the assessment of the advantages and disadvantages associated with an energy efficiency initiative (or set of initiatives) it is first necessary to characterise the set of criteria to evaluate them. This led to the consideration 
of a new subsystem (denoted 6.x in figure 3) with the following root definition:

A system used by the DM to define the set of criteria to evaluate a given energy efficiency initiative (or set of initiatives), considering his/her objectives, sensibility and relations with external entities.

The CATWOE mnemonic pertaining to this subsystem led to the following definitions:

Customer - The DM, the initiative promoter if not the same entity, external entities with relations with the DM.

Actor - The DM.

Transformation - Sensibility and Objectives of the DM $\longrightarrow$ the set of evaluation criteria.

Weltanschauung - The true interest from one initiative is only determined if all possible advantages and disadvantages are assessed.

Owner - The DM.

Environment - Measurement capability; Public Acceptability.

The activities that constitute this subsystem include the assessment of the DM's sensibility regarding which kind of initiative impacts are important (e.g. environmental, employment, health, economic), and his/her objectives (e.g. to keep energy prices low to end-users), the translation of this information into criteria, their measurability and a final decision on the criteria to use.

\section{Comparison}

The comparison was made in an informal way, by following the model and considering the equivalent actions performed under the traditional approach to initiative analysis.

Energy efficiency initiatives are currently analysed using cost-benefit or cost-effectiveness equations. The following issues are highlighted by comparing the way the various market players analyse initiatives with the conceptual model herein proposed: 
- Activities 1 and 3 of the main system are common to current practice. To be able to calculate the operands of the CBA/CEA equations it is necessary to estimate carefully all tangible costs and benefits, which requires an accurate forecast of the initiative success (adherence from end-users), including estimates of net-to-gross effects ("free-riders" and "free-drivers").

- Activity 2 is somehow considered in the European Benefit-Cost methodology, where the evaluation matrix composition is preceded by a step where the relevant perspectives are identified. The California Standard Practice establishes five relevant perspectives but in practice not all are considered in each analysis. Recently, only the societal perspective has been used, which means that activities 2 and 4 are skipped.

- In any case, the perspectives of other affected parties are only considered through a proxy, i.e., calculating what one thinks the cost-benefit or cost-effectiveness would be in that perspective, and not taking into account the actual reaction of that entity.

- The budget evaluation step is not executed in an explicit manner. It seems important that for some initiatives, under some of the perspectives, the share of the available budget taken by one initiative is a relevant criteria to be considered in the analysis.

- Regarding activity 6 , the current use of cost-benefit or cost-effectiveness equations implies that only advantages and disadvantages expressed in quantity terms (on the same unit, usually a currency unit) can be included. All other possible criteria can have an informative role only, which is not of much help to a DM. This can be improved by following an MCDA approach in activity 7, which implies a working definition of sub-system 6.x.

- Regarding this sub-system, the EUBC presents a useful approach. Its first steps are the characterisation of the market situation and the choice of relevant perspectives, followed by the selection of impacts from a glossary. In the glossary, all possible impacts are defined in terms of the relevant perspectives, and way of measuring. 
- In the framework of the proposed model, the last activity is the application of a multi-criteria method which opens the possibility of dynamically incorporating the preferences of the DM into the decision support process. In theory, these preferences might be considered during the definition of the cost-benefit or cost-effectiveness formulae as well as during the definition of the conversion formulae for impacts not naturally expressed in currency terms. However, this would be far from easy. What happens usually in current practice is the adoption of existent formulae, therefore ignoring the actual preferences of the DMs. The use of a multi-criteria method such as the ELECTRE TRI method ${ }^{12,13}$, which allows for the use of qualitative (ordinal) scales and the definition of weights in a scale independent manner, seems to be a more adequate methodological solution. Moreover, this is generally a classification problem, in which there is the need to classify one or several initiatives into pre-defined categories of interest (e.g. "not interesting”, “somewhat interesting”, "very interesting”), being the ELECTRE TRI method specially adequate for addressing this specific multi-criteria problem.

The monitoring and control of the efficacy of a Cost-Benefit analysis requires that the equation result has to be followed by a sensitivity analysis. However, the NPV calculation involves a great variety of data of different nature. This limits the number of parameters whose influence can be assessed by making them to vary, one at a time, preventing a meaningful sensitivity analysis from being carried out.

Generally, there is no control of efficiency in current practices. However, avoiding the burden of quantifying and converting impacts not naturally expressed in monetary terms to currency units is per se a way of assuring higher efficiency.

The effectiveness of the system to assure the accomplishment of its ultimate goal, the improvement of energy efficiency, can be achieved through a transparent and well documented analysis process that impels a decision. The monitoring and control can be applied to ensure this transparency. This is something which is never completely satisfied with a cost-benefit analysis, where unit conversions and compensation effects generally hide the details of analysis from the decision maker. 


\section{Debate}

Due to the nature of this study, the debate was mainly internal and ocurred during the comparison stage. However, it was necessary to assess the opinion of the actual market players regarding the change of the processes of analysis, although the absence of an immediate customer limited the scope of the debate.

To overcome this limitation, two workshops were promoted where the ideas resulting from the previous stages were discussed with representatives from some of the actual entities in the Portuguese market playing the roles specified in the rich-picture.

The results of these actions were encouraging, as it was accepted that current practices fail to address nonmonetary and non-quantifiable impacts, and that these have currently a high degree of importance, mainly due to environmental concerns, among other issues. However, it was difficult to have someone assuming the system's actor role.

The limited experience of the Portuguese energy market players with energy efficiency initiatives also hindered the depth of the debate. The few energy efficiency initiatives developed in the past were not submitted to real analysis, but were mainly policy measures. The distribution companies and the regulator are presently engaged in the first attempts to implement and evaluate DSM initiatives.

This discussion will desirably go on and other contributions, even from experts and entities outside the Portuguese market, are expected.

\section{Conclusions}

Rethinking the analysis of Energy Efficiency initiatives required a first step of problem structuring to clarify all the assumptions and to feed future developments with comprehensive and coherent information. The Soft Systems Methodology provided an effective tool for achieving this purpose, building a knowledge base from which a proposal of a methodology of analysis can be developed.

The authors are aware that this is not a "typical" application of SSM. There was not a client for the study, 
the problem-solvers are not problem-owners and the problem is not property of a single entity but can be owned by several entities. However, systems thinking helped considerably in the enquiry of the situation and to express clearly what in the beginning were only feelings of the inadequacy of current methodologies to tackle the problem.

The use of SSM resulted in a fresh view of the context and helped in the structuring of the problematic issues to be faced by distinct entities. Soft Systems Methodology was used to explore the situation, identifying stakeholders and their relations, and modelling an evaluation system which could replace the pure quantitative approach represented by cost-benefit analisys. This use of SSM to structure the problem for a multi-criteria decision making approach has the potential to be as successful as similar uses of cognitive mapping 23 .

Further developments are underway regarding the definition of relevant criteria to be used in activity 6.3 , and consequently in activities 6 and 7 of the model in figure 3

There is a need to further discuss with actual market players their views regarding relevant consequences of energy initiatives and how they should be weighed. The participants in the above mentioned workshops expressed their interest in this methodological approach and made themselves available for further discussions, and to provide answers to surveys that could lead to the definition of the set of evaluation criteria.

This definition needs a first step of characterising the Energy Efficiency initiatives, and could be the subject of a new systems study.

The inner details of activity 7 have also to be defined, although it is clear that there is a need for a multicriteria method with certain characteristics as already stated. This restricts the choice of the method to one which handles qualitative (ordinal) scales, being ELECTRE TRI one of such methods. Another issue that makes this method appropriate is that it is devoted to classify a initiative into predefined categories, which allows the analysis of isolated cases. Other multi-criteria methods which are more adequate for ranking or selecting among several alternatives could also be envisaged in such situations.

After choosing a multi-criteria method a process is needed for the definition of preference information and technical parameters, to express in a transparent manner the reasons why a DM will consider a certain initiative 
as interesting or not interesting. ELECTRE TRI requires the setting of weights for the different criteria, and thresholds of indifference, strict preference and veto for each criteria. It is also necessary to define reference initiatives to be used as boundaries of the ordered categories into which the alternatives will be classified.

The final aim of this work is to propose a comprehensive operational methodology which can be used by any of the six possible clients identified. To accomplish this, the methodology must be flexible to adapt to the different concerns of private and public entities and its results must be clear and meaningful to DMs.

Acknowledgment: This work was supported in part by the Portuguese Foundation for Science and Technology (FCT) under Grant POCTI/ESE/38422/2001.

\section{References}

${ }^{1}$ Daellenbach HG (1997). Multiple criteria decision making within Checkland's Soft Systems Methodology. In: Climaco J (ed.),Multicriteria Analysis - Proceedings of the XI International Conference on Multiple Criteria Decision Making. Springer Verlag, pp. 51-60.

2 Belton V, Ackerman F, Shepherd I (1997). Integrated support from problem structuring through to alternative evaluation using COPE and V·I.S.A. J Multi-Crit Decis Anal 6:115-130.

${ }^{3}$ Bana e Costa CA, Ensslin L, Corrêa ÉC, Vansnick JC (1999). Decision support systems in action: integrated application in a multicriteria decision aid process. Eur J Opl Res 113:315-335.

${ }^{4}$ California Public Utilities Commission and California Energy Commission (1983). Standard Practice for Cost-Benefit Analysis of Conservation and Load Management Programs.

${ }^{5}$ California Public Utilities Commission and California Energy Commission (1987). Standard Practice Manual: Economic Analysis of Demand-Side Management Programs. 
${ }^{6}$ Hirst E (1992). Definitions and tradeoffs: Cost-effectiveness of utility DSM programs. In: Summer Study on Energy Efficiency in Buildings. American Council for an Energy Efficient Economy - ACEEE, USA, pp. 8.89-8.97.

7 ELSAM (ed.) (1993). Decision Criteria on the Demand side: Integrated Resource Planning in the Danish Electric Utilities. The IRP project. ISBN 87-87090-17-1.

8 A Project Advisory Commitee and SRC International ApS (1996). European B/C Analysis Methodology (EUBC) - A Guidebook For B/C Evaluation of DSM and Energy Efficiency Services Programs. Prepared for the European Commission (DG XIIV).

9 Checkland P, Scholes J (2000). Soft Systems Methodology in Action. Wiley: Chichester.

10 Checkland P (2001). Soft systems methodology. In: Rosenhead J, Mingers J (eds.). Rational Analysis for a Problematic World Revisited. Wiley: Chichester, pp. 61-89.

11 Checkland P (1990). Systems Thinking, Systems Practice. Wiley: Chichester.

12 Yu W (1992). Aide multicritére à la decision dans le cadre de la problématique du tri: concepts, méthodes et applications. Ph.D. thesis, Université Paris-Dauphine.

13 Mousseau V, Slowinski R, Zielniewicz P (1999). A user-oriented implementation of the ELECTRE-TRI method integrating preference elicitation support. Comput Opns Res 27:757-777. 


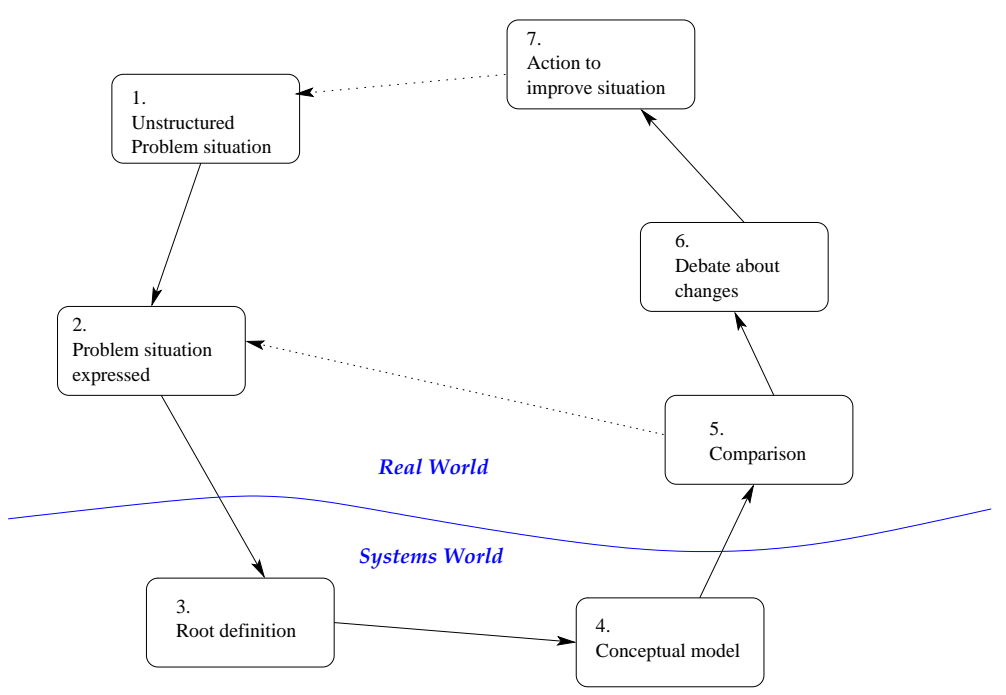

Figure 1: Seven stages of SSM 


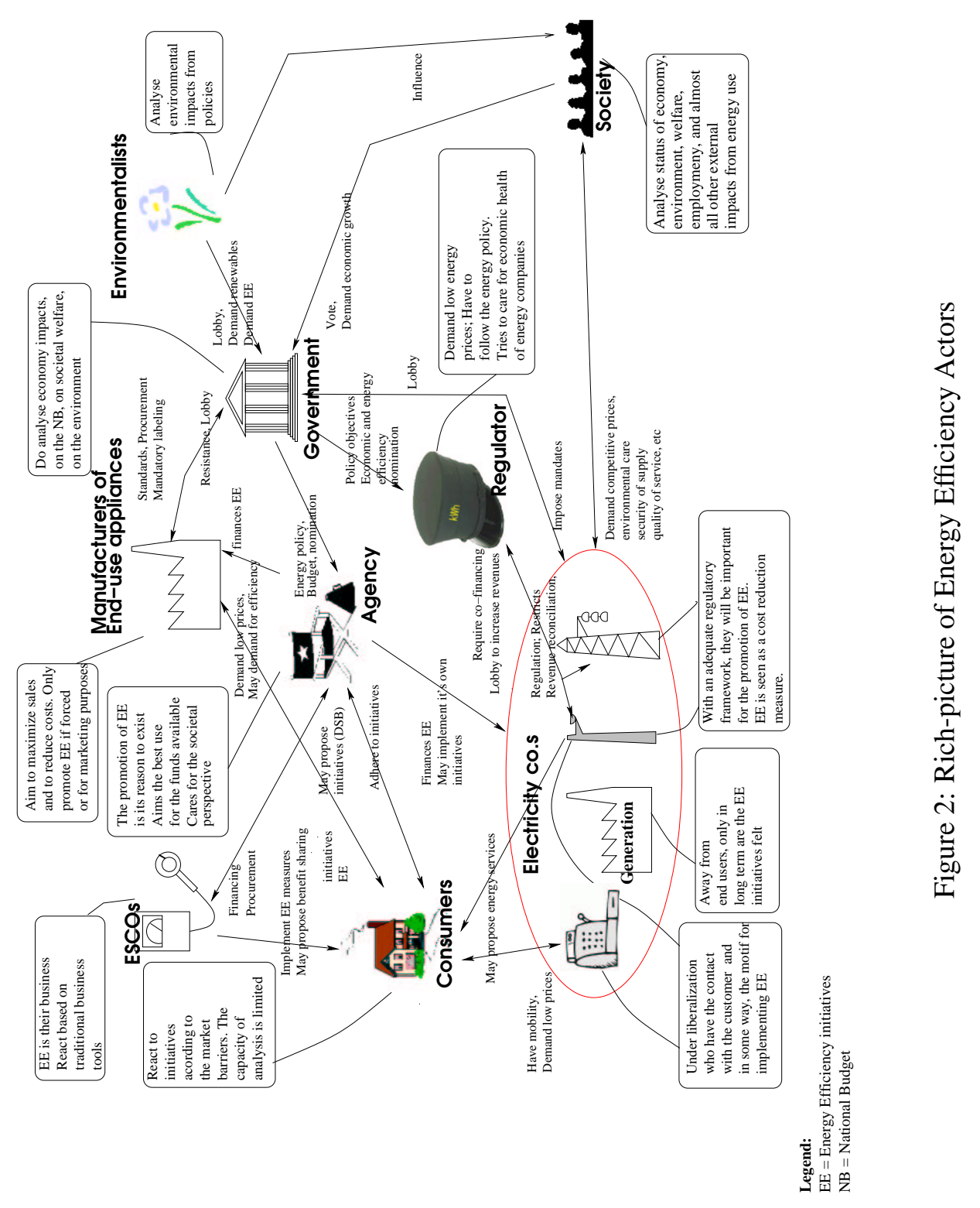




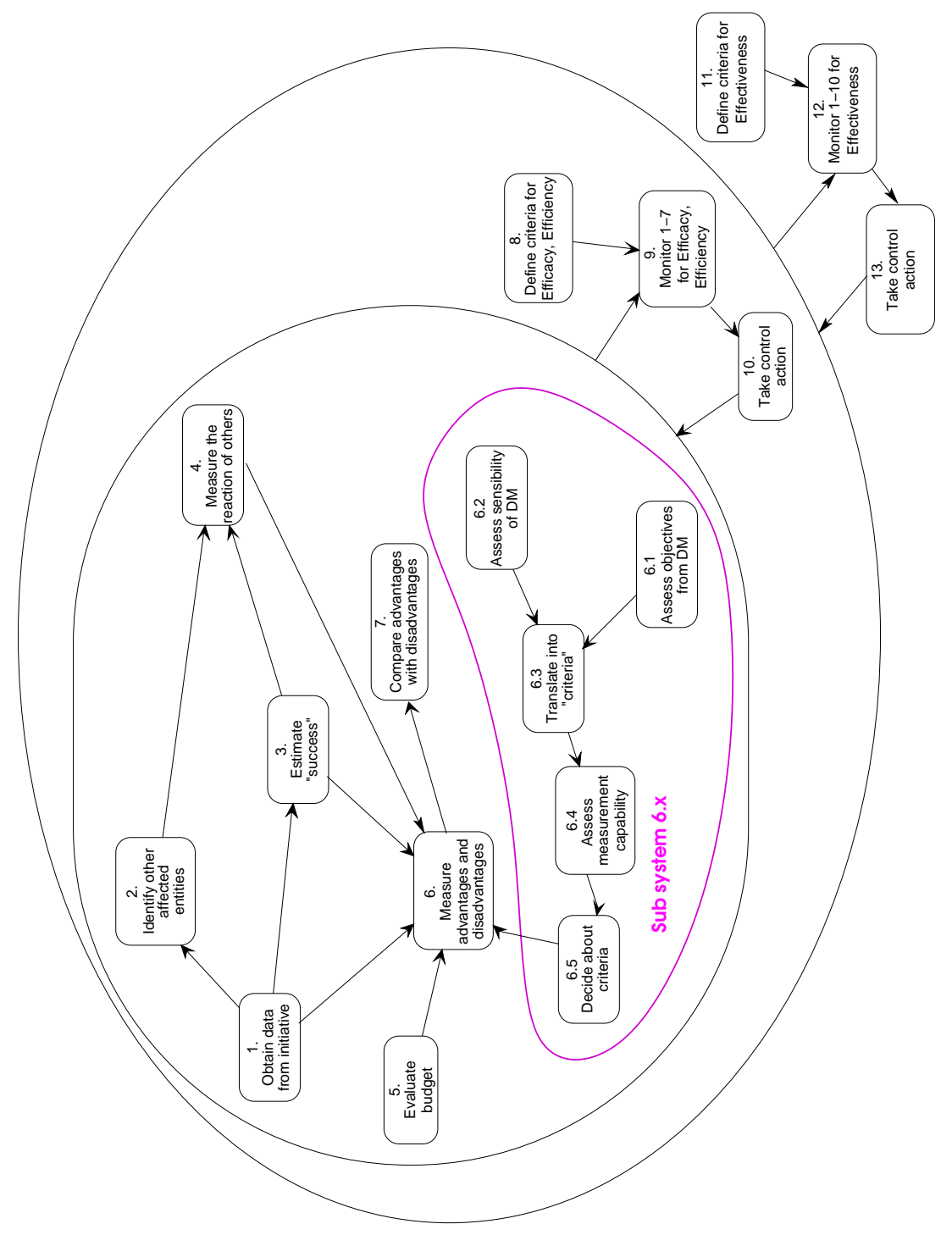

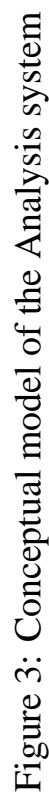

